\title{
Approximate Yang-Mills-Higgs metrics on flat Higgs bundles over an affine manifold
}

\author{
Indranil Biswas, John Loftin and Matthias Stemmler
}

\begin{abstract}
Given a flat Higgs vector bundle $(E, \nabla, \varphi)$ over a compact connected special affine manifold, we first construct a natural filtration of $E$, compatible with both $\nabla$ and $\varphi$, such that the successive quotients are polystable flat Higgs vector bundles. This is done by combining the Harder-Narasimhan filtration and the socle filtration that we construct. Using this filtration, we construct a smooth Hermitian metric $h$ on $E$ and a smooth one-parameter family $\left\{A_{t}\right\}_{t \in \mathbb{R}}$ of $C^{\infty}$ automorphisms of $E$ with the following property. Let $\nabla^{t}$ and $\varphi^{t}$ be the flat connection and flat Higgs field, respectively, on $E$ constructed from $\nabla$ and $\varphi$ using the automorphism $A_{t}$. If $\theta^{t}$ denotes the extended connection form on $E$ associated to the triple $h, \nabla^{t}$ and $\varphi^{t}$, then as $t \longrightarrow+\infty$, the connection form $\theta^{t}$ converges in the $C^{\infty}$ Fréchet topology to the extended connection form $\widehat{\theta}$ on $E$ given by the affine Yang-Mills-Higgs metrics on the polystable quotients of the successive terms in the above mentioned filtration. In particular, as $t \longrightarrow+\infty$, the curvature of $\theta^{t}$ converges in the $C^{\infty}$ Fréchet topology to the curvature of $\widehat{\theta}$.
\end{abstract}

\section{Introduction}

An affine manifold is a smooth real manifold equipped with a flat torsion-free connection $D$ on its tangent bundle [5]. An affine manifold is called special if it admits a volume form which is covariant constant with respect to this flat connection $D$. The Donaldson-Uhlenbeck-Yau correspondence, which says that a holomorphic vector bundle $V$ on a compact Kähler manifold admits a Yang-Mills metric if and only if $V$ is polystable [7, 11], extends to flat vector bundles on compact special affine manifolds [9]. In [2], flat Higgs vector bundles on affine manifolds were introduced and a DonaldsonUhlenbeck-Yau type correspondence for such bundles was established. In other words, a flat Higgs vector bundle over a compact connected special

The first-named author is supported by the J. C. Bose Fellowship. 
affine manifold admits an affine Yang-Mills-Higgs metric if and only if it is polystable.

Given a non-polystable holomorphic vector bundle $V$ on a compact Kähler manifold, it is natural to ask whether $V$ admits Hermitian metrics which are arbitrarily close to a Yang-Mills metric. A similar question can be asked in other related contexts. There is an extensive literature providing affirmative answers to this question in various contexts (see $[4,6,8,10,12]$ and references therein).

Our aim here is to address the above question for flat Higgs vector bundles over a compact connected special affine manifold. (Note this includes the case of flat vector bundles as well: simply set the Higgs field to zero.)

Let $M$ be a compact connected special affine manifold equipped with an affine Gauduchon metric $g$, and let $(E, \nabla, \varphi)$ be a flat Higgs vector bundle over $M$. The vector bundle $E$ is allowed to be real or complex.

The socle of a semistable flat Higgs vector bundle over $M$ is the unique maximal polystable flat Higgs subbundle of it (see Definition 3.6).

We first show the following (see Proposition 3.8):

Proposition 1.1. There is a unique filtration of $E$ by flat subbundles

$$
0=F_{0} \subset F_{1} \subset \cdots \subset F_{\ell-1} \subset F_{\ell}=E
$$

such that for each $i \in[1, \ell]$,

- $\varphi\left(F_{i}\right) \subset T^{*} M \otimes F_{i}$,

- $\left(F_{i} / F_{i-1}, \nabla_{i}, \varphi_{i}\right)$ is a polystable flat Higgs bundle, where $\nabla_{i}$ is the flat connection on $F_{i} / F_{i-1}$ induced by $\nabla$, and $\varphi_{i}$ is the flat Higgs field on $\left(F_{i} / F_{i-1}, \nabla_{i}\right)$ induced by $\varphi$,

- $\mu_{g}\left(F_{1}\right) \geqslant \mu_{g}\left(F_{2} / F_{1}\right) \geqslant \cdots \geqslant \mu_{g}\left(F_{i} / F_{i-1}\right) \geqslant \cdots \geqslant \mu_{g}\left(F_{\ell} / F_{\ell-1}\right)$ and

- the flat Higgs bundle $\left(F_{i} / F_{i-1}, \nabla_{i}, \varphi_{i}\right)$ is the socle of the maximal semistable subbundle of $E / F_{i-1}$ equipped with the flat connection induced by $\nabla$ and the flat Higgs field induced by $\varphi$.

The $C^{\infty}$ vector bundle $E$ is isomorphic to the direct sum

$$
\mathrm{Gr}:=\bigoplus_{i=1}^{\ell} F_{i} / F_{i-1}
$$


Fix a filtration preserving $C^{\infty}$ isomorphism $\gamma: E \longrightarrow$ Gr that induces the identity map of Gr. Denote by $\widetilde{\nabla}$ the flat connection on Gr obtained from $\nabla$ via $\gamma$, and by $\widetilde{\varphi}$ the flat Higgs field on $(\mathrm{Gr}, \widetilde{\nabla})$ obtained from $\varphi$ via $\gamma$.

Let $\nabla_{i}$ be the flat connection on $F_{i} / F_{i-1}$ induced by $\nabla$, and let $\varphi_{i}$ be the flat Higgs field on $\left(F_{i} / F_{i-1}, \nabla_{i}\right)$ induced by $\varphi$. By Theorem 2.4, each polystable flat Higgs bundle $\left(F_{i} / F_{i-1}, \nabla_{i}, \varphi_{i}\right)$ admits an affine Yang-MillsHiggs metric $h_{i}$. Let $\theta_{i}$ be the extended connection form of the Hermitian flat Higgs bundle $\left(F_{i} / F_{i-1}, \nabla_{i}, \varphi_{i}, h_{i}\right)$. Define

$$
\widehat{\theta}:=\bigoplus_{i=1}^{\ell} \theta_{i} .
$$

This form $\widehat{\theta}$ is the extended connection form on Gr equipped with the flat connection $\bigoplus_{i=1}^{\ell} \nabla_{i}$, the flat Higgs field $\bigoplus_{i=1}^{\ell} \varphi_{i}$ and the Hermitian metric $h:=\bigoplus_{i=1}^{\ell} h_{i}$.

We prove the following theorem (see Theorem 4.1):

Theorem 1.2. There is a smooth one-parameter family $\left\{A_{t}\right\}_{t \in \mathbb{R}}$ of $C^{\infty}$ automorphisms of $\mathrm{Gr}$, such that if $\nabla^{t}$ is the flat connection on $\mathrm{Gr}$ obtained from $\widetilde{\nabla}$ by the automorphism $A_{t}, \varphi^{t}$ is the flat Higgs field on $\left(\mathrm{Gr}, \nabla^{t}\right)$ obtained from $\widetilde{\varphi}$ by the automorphism $A_{t}$ and $\theta^{\varphi, t}$ is the extended connection form of $\left(\mathrm{Gr}, \nabla^{t}, \varphi^{t}, h\right)$ (both $\widetilde{\nabla}$ and $\widetilde{\varphi}$ are defined above), then as $t \longrightarrow+\infty$, the extended connection form $\theta^{\varphi, t}$ converges in the $C^{\infty}$ Fréchet topology to $\widehat{\theta}$ (see (1.1)). In particular, as $t \longrightarrow+\infty$, the extended curvature form of $\theta^{\varphi, t}$ converges to the extended curvature form of $\widehat{\theta}$ in the $C^{\infty}$ Fréchet topology.

The proof involves adapting a technique Bradlow uses to study vector bundles over Riemann surfaces [4]. The key point is that for affine manifolds, as well as for Riemann surfaces, the relevant filtration in Proposition 3.8 is via subbundles and not singular subsheaves. It may be mentioned that in [3], the results of [4] are extended to higher dimensions.

\section{Preliminaries}

Let $(M, D)$ be an affine manifold of dimension $n$, meaning $D$ is a flat torsionfree connection on the tangent bundle $T M$ of $M$. The flat connection on the cotangent bundle $T^{*} M$ induced by $D$ will be denoted by $D^{*}$.

The vector bundle of $(p, q)$ forms on $M$ is defined as

$$
\mathcal{A}^{p, q}(M):=\bigwedge^{p} T^{*} M \otimes \bigwedge^{q} T^{*} M
$$


See [9] for the definition of $\partial$ and $\bar{\partial}$ operators and the wedge product on these forms.

The affine manifold $M$ is called special if it admits a volume form $\nu$ (meaning a nowhere vanishing smooth section of $\wedge^{n} T M$ ) which is covariant constant with respect to the flat connection on $\bigwedge^{n} T M$ induced by $D$.

On a special affine manifold $(M, D, \nu)$, the volume form $\nu$ induces homomorphisms

$$
\begin{aligned}
& \mathcal{A}^{n, q}(M) \longrightarrow \bigwedge^{q} T^{*} M, \quad \nu \otimes \chi \longmapsto(-1)^{\frac{n(n-1)}{2}} \chi, \\
& \mathcal{A}^{p, n}(M) \longrightarrow \bigwedge^{p} T^{*} M, \quad \chi \otimes \nu \longmapsto(-1)^{\frac{n(n-1)}{2}} \chi ;
\end{aligned}
$$

both these homomorphisms will be called division by $\nu$. If $M$ is compact, an $(n, n)$-form $\chi$ on $M$ can be integrated by considering the integral

$$
\int_{M} \frac{\chi}{\nu}
$$

A smooth Riemannian metric $g$ on $M$ gives rise to a $(1,1)$-form $\omega_{g}$ (see $[1,9]$ ). The metric $g$ is called an affine Gauduchon metric if

$$
\partial \bar{\partial}\left(\omega_{g}^{n-1}\right)=0
$$

(recall that $n$ is the dimension of $M$ ). By Loftin [9, Theorem 5], on a compact connected special affine manifold, every conformal class of Riemannian metrics contains an affine Gauduchon metric, and this affine Gauduchon metric is unique up to multiplication by a positive constant.

Let $(E, \nabla)$ be a flat real or complex vector bundle over $M$, meaning $E$ is a smooth vector bundle and $\nabla$ is a flat connection on $E$. Given a Hermitian metric $h$ on $E$, one can define a pair

$$
\left(\partial^{h}, \bar{\partial}\right)=\left(\partial^{h, \nabla}, \bar{\partial}^{\nabla}\right)
$$

where

$$
\partial^{h, \nabla}: E \longrightarrow \mathcal{A}^{1,0}(E) \quad \text { and } \quad \bar{\partial}^{\nabla}: E \longrightarrow \mathcal{A}^{0,1}(E)
$$

are smooth differential operators. Here we write $\mathcal{A}^{p, q}(E):=\mathcal{A}^{p, q}(M) \otimes E$. This pair $\left(\partial^{h}, \bar{\partial}\right)$ is called the extended Hermitian connection of $(E, \nabla, h)$. 
Similarly, there is the locally defined extended connection form

$$
\theta \in C^{\infty}\left(\mathcal{A}^{1,0}(\operatorname{End}(E))\right)
$$

and the extended curvature form

$$
R=\bar{\partial} \theta \in C^{\infty}\left(M, \mathcal{A}^{1,1}(\operatorname{End}(E))\right)
$$

We also have the extended mean curvature

$$
K=\operatorname{tr}_{g} R \in C^{\infty}(M, \operatorname{End}(E))
$$

and the extended first Chern form

$$
c_{1}(E, h)=\operatorname{tr} R \in C^{\infty}\left(M, \mathcal{A}^{1,1}(M)\right)
$$

(see [9] for details). Here $\operatorname{tr}_{g}$ denotes contraction of differential forms using the Riemannian metric $g$ and $\operatorname{tr}$ denotes the trace map on the fibers of $\operatorname{End}(E)$.

The degree of $(E, \nabla)$ with respect to a Gauduchon metric $g$ on $M$ is defined to be

$$
\operatorname{deg}_{g}(E):=\int_{M} \frac{c_{1}(E, h) \wedge \omega_{g}^{n-1}}{\nu}
$$

it is well defined (see [9, p. 109]).

As usual, if $\operatorname{rank}(E)>0$, the slope of $E$ with respect to $g$ is defined to be

$$
\mu_{g}(E):=\frac{\operatorname{deg}_{g}(E)}{\operatorname{rank}(E)}
$$

We recall from [2]:

Definition 2.1. Let $(E, \nabla)$ be a smooth vector bundle on $M$ equipped with a flat connection. A flat Higgs field on $(E, \nabla)$ is defined to be a smooth section $\varphi$ of $T^{*} M \otimes \operatorname{End}(E)$ such that

(i) $\varphi$ is covariant constant, meaning the connection operator

$$
\widetilde{\nabla}: T^{*} M \otimes \operatorname{End}(E) \longrightarrow T^{*} M \otimes T^{*} M \otimes \operatorname{End}(E)
$$


defined by the connections $\nabla$ and $D^{*}$ on $E$ and $T^{*} M$, respectively, annihilates $\varphi$ and

(ii) $\varphi \wedge \varphi=0$.

If $\varphi$ is a flat Higgs field on $(E, \nabla)$, then $(E, \nabla, \varphi)$ is called a flat Higgs bundle.

Definition 2.2. Let $(E, \nabla, \varphi)$ be a flat Higgs bundle on $M$.

(i) $(E, \nabla, \varphi)$ is called stable (respectively, semistable) if for every flat subbundle $F$ of $E$ with $0<\operatorname{rank}(F)<\operatorname{rank}(E)$ such that $\varphi(F) \subset$ $T^{*} M \otimes F$, we have

$$
\left.\mu_{g}(F)<\mu_{g}(E) \quad \text { (respectively, } \mu_{g}(F) \leqslant \mu_{g}(E)\right)
$$

(ii) The flat Higgs bundle $(E, \nabla, \varphi)$ is called polystable if

$$
(E, \nabla, \varphi)=\bigoplus_{i=1}^{N}\left(E^{i}, \nabla^{i}, \varphi^{i}\right)
$$

where each $\left(E^{i}, \nabla^{i}, \varphi^{i}\right)$ is a stable flat Higgs bundle of slope $\mu_{g}\left(E^{i}\right)=$ $\mu_{g}(E)$.

Let $h$ be a Hermitian structure on a flat Higgs bundle $(E, \nabla, \varphi)$. The extended mean curvature $K^{\varphi}$ of $(E, \nabla, \varphi, h)$ is defined to be

$$
K^{\varphi}:=\operatorname{tr}_{g}\left(\bar{\partial} \theta+\left[\varphi, \varphi^{*}\right]\right)=K+\operatorname{tr}_{g}\left[\varphi, \varphi^{*}\right] \in \mathcal{A}^{0,0}(\text { End } E)
$$

where $\theta$ is the earlier mentioned extended connection and $K$ is defined in (2.1).

Definition 2.3. An affine Yang-Mills-Higgs metric on a flat Higgs bundle $(E, \nabla, \varphi)$ is a Hermitian metric $h$ on $E$ such that the extended mean curvature $K^{\varphi}$ of $(E, \nabla, \varphi, h)$ satisfies the equation

$$
K^{\varphi}=\gamma \operatorname{Id}_{E}
$$

for some constant scalar $\gamma$.

The following is proved in [2, Corollary 4.1]: 
Theorem 2.4. Let $(E, \nabla, \varphi)$ be a flat Higgs vector bundle over $M$. Then $E$ admits an affine Yang-Mills-Higgs metric if and only if it is polystable. Moreover, a polystable flat Higgs vector bundle over $M$ admits a unique Yang-Mills-Higgs connection.

\section{A natural filtration of flat Higgs bundles}

Let $M$ be a compact connected special affine manifold equipped with an affine Gauduchon metric $g$. In the following, by a vector bundle on $M$, we will mean either a real or a complex vector bundle.

\subsection{The Harder-Narasimhan filtration}

Lemma 3.1. Let $(E, \nabla, \varphi)$ be a flat Higgs bundle on $M$ of positive rank.

(i) There is a filtration of $E$ by subbundles

$$
0=E_{0} \subsetneq E_{1} \subsetneq \cdots \subsetneq E_{\ell-1} \subsetneq E_{\ell}=E
$$

such that for each $i \in[1, \ell]$,

- the subbundle $E_{i}$ is preserved by $\nabla$,

- $\varphi\left(E_{i}\right) \subset T^{*} M \otimes E_{i}$ and

- $\left(E_{i} / E_{i-1}, \nabla_{i}, \varphi_{i}\right)$ is a stable flat Higgs bundle, where $\nabla_{i}$ is the flat connection on $E_{i} / E_{i-1}$ induced by $\nabla$ and $\varphi_{i}$ is the flat Higgs field on $\left(E_{i} / E_{i-1}, \nabla_{i}\right)$ induced by $\varphi$.

(ii) Fix a filtration

$$
0=E_{0} \subsetneq E_{1} \subsetneq \cdots \subsetneq E_{\ell-1} \subsetneq E_{\ell}=E
$$

satisfying the conditions in (i). Let $V \subset E$ be a subbundle of positive rank such that

- $V$ is preserved by $\nabla$,

- $\varphi(V) \subset T^{*} M \otimes V$ and

- $\left(V,\left.\nabla\right|_{V},\left.\varphi\right|_{V}\right)$ is a semistable flat Higgs bundle.

Then

$$
\mu_{g}(V) \leqslant \operatorname{Max}\left(\left\{\mu_{g}\left(E_{i} / E_{i-1}\right)\right\}_{i=1}^{\ell}\right)
$$

Proof. Part (i) will be proved using induction on the rank of $E$. If $\operatorname{rank}(E)=$ 1 , then statement (i) is obvious. Assume that (i) is proved for all $E$ with $\operatorname{rank}(E) \leqslant r-1$. Now assume that $\operatorname{rank}(E)=r$. 
If the flat Higgs bundle $(E, \nabla, \varphi)$ is stable, then there is nothing to prove. If $(E, \nabla, \varphi)$ is not stable, then there is a proper subbundle $F \subsetneq E$ of positive rank such that

- $F$ is preserved by $\nabla$,

- $\varphi(F) \subset T^{*} M \otimes F$ and

- $\mu_{g}(F) \geqslant \mu_{g}(E)$.

Let $\nabla_{F}$ (respectively, $\nabla_{E / F}$ ) be the flat connection on $F$ (respectively, $E / F$ ) induced by $\nabla$, and let $\varphi_{F}$ (respectively, $\varphi_{E / F}$ ) be the flat Higgs field on $\left(F, \nabla_{F}\right)$ (respectively, $\left.\left(E / F, \nabla_{E / F}\right)\right)$ induced by $\varphi$. By the induction hypothesis, (i) holds for both $\left(F, \nabla_{F}, \varphi_{F}\right)$ and $\left(E / F, \nabla_{E / F}, \varphi_{E / F}\right)$. A filtration of $E / F$ given by (i) produces a filtration of $E$ using the natural projection $E \longrightarrow E / F$. Combined with the filtration of $F$ given by (i), this produces a filtration of $E$ satisfying the conditions in (i). This completes the proof of part (i).

To prove part (ii), note that if $\left(V_{1}, \nabla_{V_{1}}, \varphi_{V_{1}}\right)$ and $\left(V_{2}, \nabla_{V_{2}}, \varphi_{V_{2}}\right)$ are semistable flat Higgs bundles with $\mu_{g}\left(V_{1}\right)>\mu_{g}\left(V_{2}\right)$, then there is no nonzero homomorphism from $V_{1}$ to $V_{2}$ that intertwines both the flat connections and the flat Higgs fields.

Assume that

$$
\mu_{g}(V)>\mu_{g}\left(E_{i} / E_{i-1}\right)
$$

for all $i \in[1, \ell]$. Then the above observation implies that there is no non-zero homomorphism from $V$ to $E_{i} / E_{i-1}$ that intertwines both the flat connections and the flat Higgs fields. This, in turn, implies that there is no non-zero homomorphism from $V$ to $E$ that intertwines both the flat connections and the flat Higgs fields. But this contradicts the fact that $V$ is a subbundle of $E$ preserved by both $\nabla$ and $\varphi$. Therefore, we conclude that

$$
\mu_{g}(V) \leqslant \operatorname{Max}\left(\left\{\mu_{g}\left(E_{i} / E_{i-1}\right)\right\}_{i=1}^{\ell}\right) .
$$

This completes the proof of part (ii).

Let $\underline{E}:=(E, \nabla, \varphi)$ be a flat Higgs bundle on $M$ of positive rank. Define

$$
\begin{aligned}
\delta_{0}(\underline{E}):= & \operatorname{Sup}\left\{\mu_{g}(F) \mid F \text { is a non-zero flat subbundle of } E\right. \text { with } \\
& \left.\varphi(F) \subset T^{*} M \otimes F\right\} .
\end{aligned}
$$

Note that $\delta_{0}(\underline{E})<\infty$ by Lemma 3.1. 


\section{Lemma 3.2.}

1) There is a subbundle $F \subset E$ of positive rank such that

- $F$ is preserved by $\nabla$,

- $\varphi(F) \subset T^{*} M \otimes F$ and

- $\mu_{g}(F)=\delta_{0}(\underline{E})$ (defined in $\left.(3.1)\right)$.

2) There is a unique maximal subbundle $V \subset E$ satisfying all the three conditions in (1).

Proof. Fix $k \in[1, \operatorname{rank}(E)]$ such that

$$
\begin{aligned}
\delta_{0}(\underline{E})= & \operatorname{Sup}\left\{\mu_{g}(F) \mid F \text { is a flat subbundle of } E \text { of rank } k\right. \text { with } \\
& \left.\varphi(F) \subset T^{*} M \otimes F\right\} .
\end{aligned}
$$

Since $\operatorname{rank}(E)<\infty$, such a $k$ exists. Fix a point $x_{0} \in M$. Let $\operatorname{Gr}\left(k, E_{x_{0}}\right)$ be the Grassmannian parametrizing $k$-dimensional subspaces of $E_{x_{0}}$. Since $M$ is connected, a flat subbundle of $E$ of rank $k$ is uniquely determined by the point of $\operatorname{Gr}\left(k, E_{x_{0}}\right)$ given by its fiber at $x_{0}$. In other words, given any element $z \in \operatorname{Gr}\left(k, E_{x_{0}}\right)$, there is at most one flat subbundle of $E$ of rank $k$ whose fiber at $x_{0}$ gives $z$.

For each positive integer $j$, fix a point $z_{j} \in \operatorname{Gr}\left(k, E_{x_{0}}\right)$ such that

- there is a flat subbundle $F^{(j)} \subset E$ of rank $k$ satisfying the condition that the point of $\operatorname{Gr}\left(k, E_{x_{0}}\right)$ given by the fiber $F_{x_{0}}^{(j)}$ is $z_{j}$ (note that $F^{(j)}$ is unique by the above observation),

- $\varphi\left(F^{(j)}\right) \subset T^{*} M \otimes F^{(j)}$, where $F^{(j)}$ is as above and

- $\mu_{g}\left(F^{(j)}\right)>\delta_{0}(\underline{E})-\frac{1}{j}$.

Since $\operatorname{Gr}\left(k, E_{x_{0}}\right)$ is compact, the subset $\left\{z_{j}\right\}_{j=1}^{\infty}$ has an accumulation point. Let $z \in \operatorname{Gr}\left(k, E_{x_{0}}\right)$ be an accumulation point of $\left\{z_{j}\right\}_{j=1}^{\infty}$.

Let

$$
\rho: \pi_{1}\left(M, x_{0}\right) \longrightarrow \mathrm{GL}\left(E_{x_{0}}\right)
$$

be the monodromy representation for the flat connection $\nabla$ on $E$. Consider the action of $\pi_{1}\left(M, x_{0}\right)$ on $\operatorname{Gr}\left(k, E_{x_{0}}\right)$ given by $\rho$ and the standard action of $\operatorname{GL}\left(E_{x_{0}}\right)$ on $\operatorname{Gr}\left(k, E_{x_{0}}\right)$. We note that a point $z^{\prime} \in \operatorname{Gr}\left(k, E_{x_{0}}\right)$ corresponds to the fiber at $x_{0}$ of a flat subbundle of $E$ of rank $k$ if and only if $z^{\prime}$ is fixed by the action of $\rho\left(\pi_{1}\left(M, x_{0}\right)\right)$ on $\operatorname{Gr}\left(k, E_{x_{0}}\right)$.

In particular, the action of $\pi_{1}\left(M, x_{0}\right)$ on $\operatorname{Gr}\left(k, E_{x_{0}}\right)$ fixes each point $z_{j}$. From this it follows that the accumulation point $z$ is also fixed by the action 
of $\pi_{1}\left(M, x_{0}\right)$ on $\operatorname{Gr}\left(k, E_{x_{0}}\right)$. Indeed, $\pi_{1}\left(M, x_{0}\right)$ is finitely generated, and for each element $c$ of a generating set, the action of $\rho(c)$ on $\operatorname{Gr}\left(k, E_{x_{0}}\right)$ fixes $z$. Since $z$ is fixed by the action of $\pi_{1}\left(M, x_{0}\right)$ on $\operatorname{Gr}\left(k, E_{x_{0}}\right)$, there is a flat subbundle $F \subset E$ of rank $k$ such that the point of $\operatorname{Gr}\left(k, E_{x_{0}}\right)$ given by the fiber $F_{x_{0}}$ is $z$. As noted above, the flat subbundle $F$ is unique.

Since $\varphi\left(F_{x_{0}}^{(j)}\right) \subset T_{x_{0}}^{*} M \otimes F_{x_{0}}^{(j)}$ for all $j$, we conclude that

$$
\varphi\left(F_{x_{0}}\right) \subset T_{x_{0}}^{*} M \otimes F_{x_{0}} .
$$

As the section $\varphi$ is flat with respect to the connection on $T^{*} M \otimes \operatorname{End}(E)$ induced by $D^{*}$ and $\nabla$, this immediately implies that

$$
\varphi(F) \subset T^{*} M \otimes F .
$$

From the given condition that $\mu_{g}\left(F^{(j)}\right)>\delta_{0}(\underline{E})-\frac{1}{j}$ for all $j$, we conclude that $\mu_{g}(F) \geqslant \delta_{0}(\underline{E})$. But $\mu_{g}(F) \leqslant \delta_{0}(\underline{E})$ by definition of $\delta_{0}(\underline{E})$. Hence $\mu_{g}(F)=$ $\delta_{0}(\underline{E})$. This completes the proof of part (i).

To prove part (ii), let $\mathcal{C}$ be the collection of all subbundles of $E$ of positive rank that satisfy the three conditions in part (i). Take subbundles $F_{1}, F_{2} \in \mathcal{C}$. Let

$$
F_{1}+F_{2} \subset E
$$

be generated by $F_{1}$ and $F_{2}$; it is the image of $F_{1} \oplus F_{2}$ under the natural homomorphism $F_{1} \oplus F_{2} \longrightarrow E$. We will show that $F_{1}+F_{2} \in \mathcal{C}$.

Since $F_{1}$ and $F_{2}$ are flat subbundles of $E$, it follows that

- $F_{1}+F_{2}$ is also a subbundle of $E$ and

- $F_{1}+F_{2}$ is flat.

From the given condition that $\varphi\left(F_{1}\right) \subset T^{*} M \otimes F_{1}$ and $\varphi\left(F_{2}\right) \subset T^{*} M \otimes F_{2}$ it follows immediately that $\varphi\left(F_{1}+F_{2}\right) \subset T^{*} M \otimes\left(F_{1}+F_{2}\right)$. Therefore,

$$
\mu_{g}\left(F_{1}+F_{2}\right) \leqslant \delta_{0}(\underline{E}) .
$$

We will show that

$$
\mu_{g}\left(F_{1}+F_{2}\right)=\delta_{0}(\underline{E}) .
$$

Since $F_{1}$ and $F_{2}$ are flat subbundles of $E$, the intersection $F_{1} \cap F_{2}$ is also a flat subbundle of $E$. We have a short exact sequence of flat bundles

$$
0 \longrightarrow F_{1} \cap F_{2} \longrightarrow F_{1} \oplus F_{2} \longrightarrow F_{1}+F_{2} \longrightarrow 0,
$$


where $F_{1} \oplus F_{2}$ is equipped with the flat connection $\left.\left.\nabla\right|_{F_{1}} \oplus \nabla\right|_{F_{2}}$.

If $F_{1} \cap F_{2}$ is of rank zero, then $\mu_{g}\left(F_{1}+F_{2}\right)=\mu_{g}\left(F_{1} \oplus F_{2}\right)=\delta_{0}(\underline{E})$, because $\mu_{g}\left(F_{1}\right)=\delta_{0}(\underline{E})=\mu_{g}\left(F_{2}\right)$. So $(3.3)$ holds if the rank of $F_{1} \cap F_{2}$ is zero. Therefore, assume that the rank of $F_{1} \cap F_{2}$ is positive.

From (3.4) we have

$$
\mu_{g}\left(F_{1} \oplus F_{2}\right)=\frac{\mu_{g}\left(F_{1} \cap F_{2}\right) \operatorname{rank}\left(F_{1} \cap F_{2}\right)+\mu_{g}\left(F_{1}+F_{2}\right) \operatorname{rank}\left(F_{1}+F_{2}\right)}{\operatorname{rank}\left(F_{1} \cap F_{2}\right)+\operatorname{rank}\left(F_{1}+F_{2}\right)} .
$$

Note that $\varphi\left(F_{1} \cap F_{2}\right) \subset T^{*} M \otimes\left(F_{1} \cap F_{2}\right)$, because $\varphi\left(F_{i}\right) \subset T^{*} M \otimes F_{i}, i=$ 1, 2. Hence

$$
\mu_{g}\left(F_{1} \cap F_{2}\right) \leqslant \delta_{0}(\underline{E}) .
$$

Since $\mu_{g}\left(F_{1} \oplus F_{2}\right)=\delta_{0}(\underline{E})$, in view of (3.2) and (3.6), from (3.5) we conclude that

$$
\mu_{g}\left(F_{1}+F_{2}\right)=\delta_{0}(\underline{E})=\mu_{g}\left(F_{1} \cap F_{2}\right)
$$

Therefore, the subbundle $F_{1}+F_{2} \in \mathcal{C}$.

Since the rank of $E$ is finite, this implies that $V \subset E$ generated by all subbundles in the family $\mathcal{C}$ also lies in $\mathcal{C}$. This completes the proof of the lemma.

Consider the unique maximal subbundle $V$ of $E$ in Lemma 3.2. Since $\mu_{g}(V)=\delta_{0}(\underline{E})$, it follows that $\underline{V}:=\left(V,\left.\nabla\right|_{V},\left.\varphi\right|_{V}\right)$ is semistable. This flat Higgs bundle $\underline{V}$ will be called the maximal semistable subbundle of $\underline{E}$.

Lemma 3.2 has the following corollary:

Corollary 3.3. Let $(E, \nabla, \varphi)$ be a flat Higgs bundle on $M$ of positive rank. There is a unique filtration of $E$ by subbundles

$$
0=F_{0} \subsetneq F_{1} \subsetneq \cdots \subsetneq F_{\ell-1} \subsetneq F_{\ell}=E
$$

such that for each $i \in[1, \ell]$,

- the subbundle $F_{i}$ is preserved by $\nabla$,

- $\varphi\left(F_{i}\right) \subset T^{*} M \otimes F_{i}$,

- $\left(F_{i} / F_{i-1}, \nabla_{i}, \varphi_{i}\right)$ is a semistable flat Higgs bundle, where $\nabla_{i}$ is the flat connection on $F_{i} / F_{i-1}$ induced by $\nabla$ and $\varphi_{i}$ is the flat Higgs field on $\left(F_{i} / F_{i-1}, \nabla_{i}\right)$ induced by $\varphi$ and 
- $\mu_{g}\left(F_{1}\right)>\mu_{g}\left(F_{2} / F_{1}\right)>\cdots>\mu_{g}\left(F_{i} / F_{i-1}\right)>\cdots>\mu_{g}\left(F_{\ell} / F_{\ell-1}\right)$.

Proof. For each $i \in[1, \ell]$, the quotient $F_{i} / F_{i-1}$ equipped with the flat connection induced by $\nabla$ and the flat Higgs field induced by $\varphi$ is the maximal semistable subbundle of $E / F_{i-1}$ equipped with the flat connection induced by $\nabla$ and the flat Higgs field induced by $\varphi$.

\subsection{The socle filtration}

Let $\underline{E}:=(E, \nabla, \varphi)$ be a semistable flat Higgs bundle over $M$ of positive rank.

Let $F_{1}$ and $F_{2}$ be two flat subbundles of $E$ of positive rank such that for $i=1,2$,

- $\varphi\left(F_{i}\right) \subset T^{*} M \otimes F_{i}$,

- $\mu_{g}\left(F_{i}\right)=\mu_{g}(E)$ and

- the flat bundle $F_{i}$ equipped with the flat Higgs field $\left.\varphi\right|_{F_{i}}$ is polystable.

Let

$$
F_{1}+F_{2} \subset E
$$

be generated by $F_{1}$ and $F_{2}$. We saw in the proof of Lemma 3.2 that $F_{1}+F_{2}$ is a flat subbundle of $E$, and $\varphi\left(F_{1}+F_{2}\right) \subset T^{*} M \otimes\left(F_{1}+F_{2}\right)$.

Proposition 3.4. The following two hold:

1) $\mu_{g}\left(F_{1}+F_{2}\right)=\mu_{g}(E)$ and

2) the flat vector bundle $F_{1}+F_{2}$ equipped with the flat Higgs field $\left.\varphi\right|_{F_{1}+F_{2}}$ is polystable.

Proof. The first statement is already proved; see (3.7). Note that $\delta_{0}(\underline{E})=$ $\mu_{g}(E)$ because $(E, \nabla, \varphi)$ is semistable.

To prove the second statement, first note that (3.4) is in fact a short exact sequence of flat Higgs bundles (all the Higgs fields are induced by $\varphi$ ). We have $\mu_{g}\left(F_{1} \cap F_{2}\right)=\mu_{g}(E)$ (see $(3.7)$ ). The flat vector bundle $F_{1} \oplus F_{2}$ equipped with the Higgs field $\left.\left.\varphi\right|_{F_{1}} \oplus \varphi\right|_{F_{2}}$ is polystable of slope $\mu_{g}(E)$. Hence $\left(F_{1} \cap F_{2},\left.\nabla\right|_{F_{1} \cap F_{2}},\left.\varphi\right|_{F_{1} \cap F_{2}}\right)$ is also polystable. 
Writing the flat polystable Higgs bundle $\left(F_{1} \oplus F_{2},\left.\left.\nabla\right|_{F_{1}} \oplus \nabla\right|_{F_{2}},\left.\varphi\right|_{F_{1}} \oplus\right.$ $\left.\left.\varphi\right|_{F_{2}}\right)$ as a direct sum of stable Higgs bundles, we conclude that it is isomorphic to the direct sum of flat Higgs bundles

$$
\left(F_{1} \cap F_{2},\left.\nabla\right|_{F_{1} \cap F_{2}},\left.\varphi\right|_{F_{1} \cap F_{2}}\right) \oplus\left(F_{1}+F_{2},\left.\nabla\right|_{F_{1}+F_{2}},\left.\varphi\right|_{F_{1}+F_{2}}\right)
$$

(in other words, the short exact sequence of flat Higgs bundles given by (3.4) splits). Therefore, the last statement of the proposition is proved.

Corollary 3.5. There is a unique maximal flat subbundle $F$ of E such that

- $\varphi(F) \subset T^{*} M \otimes F$,

- $\mu_{g}(F)=\mu_{g}(E)$ and

- the flat bundle $F$ equipped with the flat Higgs field $\left.\varphi\right|_{F}$ is polystable.

Proof. Let $F \subset E$ be generated by all flat subbundles of $E$ satisfying the three conditions in the corollary. From Proposition 3.4 we conclude that $F$ is a flat subbundle of $E$ satisfying the three conditions in the corollary.

Definition 3.6. If $F$ is the unique maximal flat subbundle of $E$ satisfying the conditions in Corollary 3.5, then the polystable flat Higgs bundle $\underline{F}:=$ $\left(F,\left.\nabla\right|_{F},\left.\varphi\right|_{F}\right)$ is called the socle of $\underline{E}$.

Corollary 3.7. Let $\underline{E}:=(E, \nabla, \varphi)$ be a semistable flat Higgs bundle over $M$ of positive rank. There is a unique filtration of $E$ by flat subbundles

$$
0=F_{0} \subset F_{1} \subset \cdots \subset F_{m-1} \subset F_{m}=E
$$

such that for each $j \in[1, m]$,

- $\varphi\left(F_{j}\right) \subset T^{*} M \otimes F_{j}$ and

- the flat subbundle $F_{j} / F_{j-1} \subset E / F_{j-1}$ equipped with the flat Higgs field induced by $\varphi$ is the socle of the flat semistable vector bundle $E / F_{j-1}$ equipped with the flat Higgs field induced by $\varphi$.

\subsection{The combined filtration}

Let $\underline{E}:=(E, \nabla, \varphi)$ be a flat Higgs bundle over $M$ of positive rank. Let

$$
0=F_{0} \subsetneq F_{1} \subsetneq \cdots \subsetneq F_{\ell-1} \subsetneq F_{\ell}=E
$$


be the unique filtration of $E$ given by Corollary 3.3. For each $i \in[1, \ell]$, consider the quotient $F_{i} / F_{i-1}$ equipped with the flat connection induced by $\nabla$ and the flat Higgs field induced by $\varphi$. Since it is semistable, $F_{i} / F_{i-1}$ has a unique filtration given by Corollary 3.7. These filtrations together produce a unified filtration of $E$ as follows.

Proposition 3.8. There is a unique filtration of $E$ by flat subbundles

$$
0=F_{0} \subset F_{1} \subset \cdots \subset F_{\ell-1} \subset F_{\ell}=E
$$

such that for each $i \in[1, \ell]$,

- $\varphi\left(F_{i}\right) \subset T^{*} M \otimes F_{i}$,

- $\left(F_{i} / F_{i-1}, \nabla_{i}, \varphi_{i}\right)$ is a polystable flat Higgs bundle, where $\nabla_{i}$ is the flat connection on $F_{i} / F_{i-1}$ induced by $\nabla$ and $\varphi_{i}$ is the flat Higgs field on $\left(F_{i} / F_{i-1}, \nabla_{i}\right)$ induced by $\varphi$,

- $\mu_{g}\left(F_{1}\right) \geqslant \mu_{g}\left(F_{2} / F_{1}\right) \geqslant \cdots \geqslant \mu_{g}\left(F_{i} / F_{i-1}\right) \geqslant \cdots \geqslant \mu_{g}\left(F_{\ell} / F_{\ell-1}\right)$ and

- the flat Higgs bundle $\left(F_{i} / F_{i-1}, \nabla_{i}, \varphi_{i}\right)$ is the socle of the maximal semistable subbundle of $E / F_{i-1}$ equipped with the flat connection induced by $\nabla$ and the flat Higgs field induced by $\varphi$.

\section{Flow under a one-parameter group of automorphisms}

As before, let $M$ be a compact connected special affine manifold equipped with an affine Gauduchon metric $g$. Let $(E, \nabla, \varphi)$ be a flat Higgs bundle over $M$. Let

$$
0=F_{0} \subset F_{1} \subset \cdots \subset F_{\ell-1} \subset F_{\ell}=E
$$

be the filtration constructed in Proposition 3.8.

Fix $C^{\infty}$ subbundles $V_{i} \subset E, 0 \leqslant i \leqslant \ell$, such that

(1) the natural homomorphism

$$
\gamma: \bigoplus_{i=0}^{\ell} V_{i} \longrightarrow E
$$

is an isomorphism and 
(2) for every $j \in[0, \ell]$,

$$
\gamma\left(\bigoplus_{i=0}^{j} V_{i}\right)=F_{j} .
$$

Note that $V_{0}=0$, and the composition

$$
V_{i} \longleftrightarrow F_{i} \longrightarrow F_{i} / F_{i-1}
$$

is an isomorphism for all $i \in[1, \ell]$.

Let $\nabla_{i}$ be the flat connection on $V_{i}$ induced by $\nabla$ using the isomorphism of $V_{i}$ with $F_{i} / F_{i-1}$ in (4.2). Let $\varphi_{i}$ be the flat Higgs field on $\left(V_{i}, \nabla_{i}\right)$ induced by $\varphi$ using the isomorphism in (4.2). Let $\widetilde{\nabla}$ be the flat connection on $\bigoplus_{i=1}^{\ell} V_{i}$ given by $\nabla$ using the isomorphism $\gamma$. Let $\widetilde{\varphi}$ be the flat Higgs field on $\left(\bigoplus_{i=1}^{\ell} V_{i}, \widetilde{\nabla}\right)$ given by $\varphi$ using the isomorphism $\gamma$.

Each of the polystable flat Higgs bundles $\left(V_{i}, \nabla_{i}, \varphi_{i}\right)$ is equipped with an affine Yang-Mills-Higgs metric $h_{i}$ (Theorem 2.4). Let

$$
h:=\bigoplus_{i=1}^{\ell} h_{i}
$$

be the induced Hermitian metric on $\bigoplus_{i=1}^{\ell} V_{i}$. Let

$$
\widehat{\theta}
$$

denote the extended connection form on the bundle $\bigoplus_{i=1}^{\ell} V_{i}$ equipped with the flat connection $\bigoplus_{i=1}^{\ell} \nabla_{i}$, the flat Higgs field $\bigoplus_{i=1}^{\ell} \varphi_{i}$ and the Hermitian metric $h$. Note that $\widehat{\theta}$ is the direct sum of the extended connection forms on $\left(V_{i}, \nabla_{i}, \varphi_{i}\right)$ associated to the affine Yang-Mills-Higgs metrics $h_{i}$.

For every $t \in \mathbb{R}$, define a $C^{\infty}$ automorphism of $\bigoplus_{i=1}^{\ell} V_{i}$

$$
A_{t}:=\bigoplus_{j=1}^{\ell} \exp (j t) \operatorname{Id}_{V_{j}} .
$$

Define $\nabla^{t}:=A_{t} \circ \widetilde{\nabla} \circ A_{t}^{-1}$ and $\varphi^{t}:=A_{t} \circ \widetilde{\varphi} \circ A_{t}^{-1}$. In particular, the flat Higgs bundle $\left(\bigoplus_{i=1}^{\ell} V_{i}, \nabla^{t}, \varphi^{t}\right)$ is isomorphic to the flat Higgs bundle $\left(\bigoplus_{i=1}^{\ell} V_{i}, \widetilde{\nabla}, \widetilde{\varphi}\right)$ for all $t$. Hence $\left(\bigoplus_{i=1}^{\ell} V_{i}, \nabla^{t}, \varphi^{t}\right)$ is isomorphic to $(E, \nabla, \varphi)$ for all $t$.

Keep the Hermitian metric $h$ on $\bigoplus_{i=1}^{\ell} V_{i}$ fixed, meaning it is not changed as $t$ changes. Let $\theta^{t}$ be the extended connection form on the bundle $\bigoplus_{i=1}^{\ell} V_{i}$ 
equipped with the flat connection $\nabla^{t}$ and the Hermitian metric $h$. Let

$$
\theta^{\varphi, t}:=\left(\theta^{t}+\varphi^{t},\left(\varphi^{t}\right)^{*}\right)
$$

be the extended connection form on $\left(\bigoplus_{i=1}^{\ell} V_{i}, \nabla^{t}, \varphi^{t}, h\right)$; here $\left(\varphi^{t}\right)^{*}$ denotes the adjoint of $\varphi^{t}$ with respect to $h$. (See $[2$, p. 6].)

Theorem 4.1. As $t \longrightarrow+\infty$, the extended connection form $\theta^{\varphi, t}$ in (4.5) converges in the $C^{\infty}$ Fréchet topology to the extended connection form $\widehat{\theta}$ in (4.3). Therefore, as $t \longrightarrow+\infty$, the extended curvature form of $\theta^{\varphi, t}$ converges in the $C^{\infty}$ Fréchet topology to the extended curvature form of $\widehat{\theta}$.

Proof. For notational convenience, we only give a proof for the case where $\ell=2$. The proof for filtrations of arbitrary length works analogously.

Since the Hermitian metric $h$ on $V_{1} \oplus V_{2}$ is kept fixed, to prove the theorem it suffices to show that

- as $t \longrightarrow+\infty$, the flat connection $\nabla^{t}$ converges in the $C^{\infty}$ Fréchet topology to $\nabla_{1} \oplus \nabla_{2}$ and

- as $t \longrightarrow+\infty$, the Higgs field $\varphi^{t}$ converges in the $C^{\infty}$ Fréchet topology to $\varphi_{1} \oplus \varphi_{2}$.

In terms of the direct sum decomposition $V_{1} \oplus V_{2}$, we have

$$
\widetilde{\nabla}=\left(\begin{array}{cc}
\nabla_{1} & \alpha \\
0 & \nabla_{2}
\end{array}\right)
$$

where $\alpha$ is a smooth complex one-form on $M$ with values in $\operatorname{Hom}\left(V_{1}, V_{2}\right)$. From the definition of $A_{t}$ (see (4.4)), we obtain

$$
\nabla^{t}=\left(\begin{array}{cc}
\nabla_{1} & \exp (-t) \cdot \alpha \\
0 & \nabla_{2}
\end{array}\right)
$$

This implies that as $t \longrightarrow+\infty$, the flat connection $\nabla^{t}$ converges in the $C^{\infty}$ Fréchet topology to $\nabla_{1} \oplus \nabla_{2}$.

Analogously, we have

$$
\widetilde{\varphi}=\left(\begin{array}{cc}
\varphi_{1} & \beta \\
0 & \varphi_{2}
\end{array}\right)
$$

where $\beta$ is a smooth section of $\mathcal{A}^{1,0}\left(\operatorname{Hom}\left(V_{2}, V_{1}\right)\right)$. It follows that

$$
\varphi^{t}=A_{t} \circ \widetilde{\varphi} \circ A_{t}^{-1}=\left(\begin{array}{cc}
\varphi_{1} & \exp (-t) \cdot \beta \\
0 & \varphi_{2}
\end{array}\right)
$$


implying that as $t \longrightarrow+\infty$, the Higgs field $\varphi^{t}$ converges in the $C^{\infty}$ Fréchet topology to $\varphi_{1} \oplus \varphi_{2}$. This completes the proof.

\section{References}

[1] I. Biswas and J. Loftin, Hermitian-Einstein connections on principal bundles over flat affine manifolds, Int. J. Math. 23(4) (2012), doi:10.1142/S0129167X12500395.

[2] I. Biswas, J. Loftin and M. Stemmler, Affine Yang-Mills-Higgs metrics, J. Symp. Geom. 11 (2013), 377-404.

[3] I. Biswas, S.B. Bradlow, A. Jacob and M. Stemmler, Automorphisms and connections on Higgs bundles over compact Kähler manifolds, Diff. Geom. Appl. 32 (2014), 139-152.

[4] S.B. Bradlow, Hermitian-Einstein inequalities and Harder-Narasimhan filtrations, Int. J. Math. 6 (1995), 645-656.

[5] S.-Y. Cheng and S.-T. Yau, The real Monge-Ampère equation and affine flat structures, Proceedings of the 1980 Beijing Symposium on Differential Geometry and Differential Equations, 1-3 (Beijing, 1980), Science Press, Beijing, 1982, 339-370.

[6] G.D. Daskalopoulos and R.A. Wentworth, Convergence properties of the Yang-Mills flow on Kähler surfaces, J. Reine Angew. Math. 575 (2004), 69-99.

[7] S.K. Donaldson, Infinite determinants, stable bundles and curvature, Duke Math. J. 54 (1987), 231-247.

[8] M.-C. Hong and G. Tian, Asymptotical behaviour of the Yang-Mills flow and singular Yang-Mills connections, Math. Ann. 330 (2004), 441-472.

[9] J. Loftin, Affine Hermitian-Einstein metrics, Asian J. Math. 13 (2009), 101-130.

[10] J. Råde, On the Yang-Mills heat equation in two and three dimensions, J. Reine Angew. Math. 431 (1992), 123-163.

[11] K. Uhlenbeck and S.-T. Yau, On the existence of Hermitian-YangMills connections in stable vector bundles, Comm. Pure Appl. Math. 39 (1986), 257-293. 
[12] G. Wilkin, Morse theory for the space of Higgs bundles, Comm. Anal.

Geom. 16 (2008), 283-332.

School of Mathematics

Tata Institute of Fundamental Research

Homi BHabHa RoAD, Bombay 400005

INDIA

E-mail address: indranil@math.tifr.res.in

Department of Mathematics and Computer Science

Rutgers UNIVERSITY AT NEWARK

NEWARK, NJ 07102

USA

E-mail address: loftin@rutgers.edu

FACHBEREICH MATHEMATIK UND INFORMATIK

PhilipPs-Universität MARBURG

HANS-MEERWEIN-STRASSE

LAHNBERGE, 35032 MARburg

GERMANY

E-mail address: stemmler@mathematik.uni-marburg.de 